\title{
Structural determinants of PINK1 topology and dual subcellular distribution
}

\author{
William Lin, Un Jung Kang*
}

\begin{abstract}
Background: PINK1 is a mitochondria-targeted kinase that constitutively localizes to both the mitochondria and the cytosol. The mechanism of how PINK1 achieves cytosolic localization following mitochondrial processing remains unknown. Understanding PINK1 subcellular localization will give us insights into PINK1 functions and how mutations in PINK1 lead to Parkinson's disease. We asked how the mitochondrial localization signal, the transmembrane domain, and the kinase domain participate in PINK1 localization.

Results: We confirmed that PINK1 mitochondrial targeting signal is responsible for mitochondrial localization. Once inside the mitochondria, we found that both PINK1 transmembrane and kinase domain are important for membrane tethering and cytosolic-facing topology. We also showed that PINK1 dual subcellular distribution requires both Hsp90 interaction with the kinase domain and the proteolysis at a cleavage site downstream of the transmembrane domain because removal of this cleavage site completely abolished cytosolic PINK1. In addition, the disruption of the Hsp90-PINK1 interaction increased mitochondrial PINK1 level.

Conclusion: Together, we believe that once PINK1 enters the mitochondria, PINK1 adopts a tethered topology because the transmembrane domain and the kinase domain prevent PINK1 forward movement into the mitochondria. Subsequent proteolysis downstream of the transmembrane domain then releases PINK1 for retrograde movement while PINK1 kinase domain interacts with Hsp90 chaperone. The significance of this dual localization could mean that PINK1 has compartmental-specific functions.
\end{abstract}

\section{Background}

Nuclear-encoded mitochondrial proteins synthesized in the cytosol are targeted to the mitochondria by one of two types of targeting signals, a hydrophobic presequence (MLS) and/or a cryptic internal sequence [1]. The MLS directs the precursor protein to the translocase of the outer membrane (TOMM) where translocation begins. In addition, the MLS affects the precursor import efficiency as determined by the length of signal peptide [2] and encodes the submitochondrial localization of mitochondrial proteins after mitochondrial processing, as exemplified by the presence of a cleavable or non-cleavable stop-transfer signal [3]. Redistribution after mitochondrial processing can also be affected by protein folding, even though most precursor translocation requires unfolding. Of the two reported examples of protein folding affecting mitochondrial import, the

\footnotetext{
* Correspondence: unkang@uchicago.edu
Department of Neurology, University of Chicago Medical Center, Chicago,

* Correspondence: unkang@uchicago.edu
Department of Neurology, University of Chicago Medical Center, Chicago, Illinois 60637, USA
} (1)

propeller domain of $\mathrm{PP} 2 \mathrm{~A} / \mathrm{B} \beta 2$ subunit arrests the import process and becomes on OMM protein [4] whereas rapid folding of yeast fumarase during the import favors the retrograde movement for a cytosolic localization [5]. Interestingly, there are only a handful of proteins that distribute between the mitochondria and cytosol in a constitutive manner, fumarase being the most studied example. It has been demonstrated that fumarase has a $30 \% / 70 \%$ mitochondria/cytosol isoprotein distribution and this dual localization occurs after mitochondrial processing [6].

The PINK1 gene encodes a kinase protein that contains an N-terminal MLS and mutations in PINK1 are linked to a recessive form of Parkinson's disease. Using a heterologous expression system, varying lengths of PINK1 MLS were tested (1-33aa, 1-77aa, and 1-156aa) and all PINK1 MLS-GFP fusion proteins co-localized with mitochondrial markers, such as mitotracker or TOM20 fluorescence [7-9]. These studies proved that PINK1 MLS is sufficient for mitochondrial targeting. 
The submitochondrial localization of PINK1, by biochemical fractionation, shows that all forms of PINK1 are found at the outer membrane, intermembrane space, and inner membrane, but not the matrix $[8,10]$. However, the subcellular localization of endogenous and overexpressed PINK1 in cell culture models show that PINK1 does not solely localize to the mitochondrial fraction, as cytosolic and microsomal fractions are found to contain all cleaved forms of PINK1 [7,11-13]. Overexpression of cytosolic PINK1, one that lacks the MLS, exhibits protective function against MPTP toxicity in mice and in cell culture [14]. Also, proteins found to associate with PINK1 are either cytosolic (Parkin, DJ-1, Hsp90, and Cdc37 $[12,13,15,16])$ or cytosolically exposed (Miro and Milton [17]). Only HtrA2 and TRAP1 are found to associate with PINK1 in the mitochondria $[10,18]$. Currently no studies have examined the function of the mitochondrial form of PINK1 in the absence of the cytosolic PINK1.

Several important questions arise from PINK1 dual localization: what purpose does the PINK1 MLS serve if a functional PINK1 protein is also found in the cytosol? How does PINK1 redistribute after mitochondrial processing? Is the function of PINK1 different in mitochondria as compared to the cytosol? We are very interested to understand the mechanism behind PINK1 dual distribution, especially given the evidence that the mitochondrial pool of PINK1 is tethered to the OMM (with the kinase domain exposed to the cytosol) and removal of the PINK1 transmembrane domain mislocalizes PINK1 inside the mitochondria [19]. We previously showed that PINK1 cleaved forms are generated from the mitochondrial processing of PINK1 precursor, thus suggesting that PINK1 cytosolic redistribution occurs after cleavage [12]. We hypothesize that while the PINK1 MLS can direct proteins to the mitochondria, the required interaction between the PINK1 kinase domain and Hsp90 chaperone favors a retrograde movement, thus resulting in a cytosolic localization. To test our hypothesis, we fused wildtype PINK1 as well as PINK1 mutant that lacks Hsp90 chaperone interaction with other known MLS and examined the cytosolic and mitochondrial distribution of these proteins when expressed in a cell culture model.

\section{Results \\ PINK1 N-terminal cleavages occur before and after PINK1 transmembrane domain}

At first glance, PINK1 MLS is similar either to those of inner membrane (uncleaved transmembrane anchor) or intermembrane space proteins (bipartite presequence). The difference between these two signals is the cleavage site after the transmembrane domain, which would determine whether or not the protein is anchored.
Overexpression of WT PINK1 in cell lines leads to the generation of three or more PINK1 forms, suggesting the presence of multiple cleavage sites $[7,9,11,12]$. The pattern of endogenous PINK1 protein is debatable due to low endogenous PINK1 expression and the lack of a high affinity antibody although it is generally accepted and agreed upon that at least two endogenous PINK1 bands are detectable-the full length and a cleaved form around $55 \mathrm{kDa}[13,20,21]$. A most recent paper showed three endogenous bands [21]. We and others have previously demonstrated that endogenous PINK1 behaves similarly to the overexpressed PINK1 counterparts in that PINK1 FL accumulates under valinomycin treatment and PINK1 $\Delta 1$ and $\Delta 2$ accumulate under proteasome inhibitor treatment $[9,12,22]$. Using these two chemical inhibitors, we first wanted to establish that Hela cells express three forms of endogenous PINK1. We observed that valinomycin treatment led to the increase of PINK1 FL, and epoxomicin treatment increased two lower protein bands when compared to untreated cells (Figure 1A). With epoxomicin, the heavily accumulated protein is PINK1 $\Delta 1$ and the protein around $45 \mathrm{kDa}$ is the PINK1 $\Delta 2$ form. We also tested the specificity of these three PINK1 bands by using siRNA to knockdown endogenous PINK1. In two independent siPINK1 transfections, western blot showed all three endogenous PINK1 proteins were decreased (Figure 1A'), confirming the hypothesis that endogenous PINK1 also expresses two cleaved forms. In addition, we do not believe that the PINK1 $\Delta 2$ form is a mere degradation product because our previous metabolic labeling data showed that PINK1 $\Delta 2$ form is most stable protein of all PINK1 forms [12].

Potential mitochondrial processing motifs have been examined for PINK1 MLS, where one predicted site is mapped at amino acid 35 and the second site around amino acids 77 [8]. Both predicted cleavage sites correspond with the consensus R-2 or R-10 matrix processing motif [23]. The second processing consensus motif is upstream of the PINK1 transmembrane domain (predicted from amino acids 90-110) and proteolysis at this site can generate a protein with similar molecular weight to PINK1 $\Delta 1$ form. We were first interested in determining the approximate molecular sizes of each PINK1 cleaved products, which might yield clues about possible proteolytic sites. We constructed and expressed $\mathrm{N}$-terminal serial truncation mutants, $\Delta 35$ PINK1, $\Delta 70$ PINK1, $\Delta 105$ PINK1, and $\Delta 151$ PINK1. By western blot, $\Delta 70$ and $\Delta 105$ PINK1 showed proteins expressed as similar molecular weight as WT PINK1 $\Delta 1$ and $\Delta 2$ cleaved products (Figure $1 \mathrm{C}$ ). We also observed that $\Delta 151$ PINK1 was only expressed as a single form, corresponding to the smallest band in all of the PINK1 constructs (Figure 1C). Data from these truncation mutants 


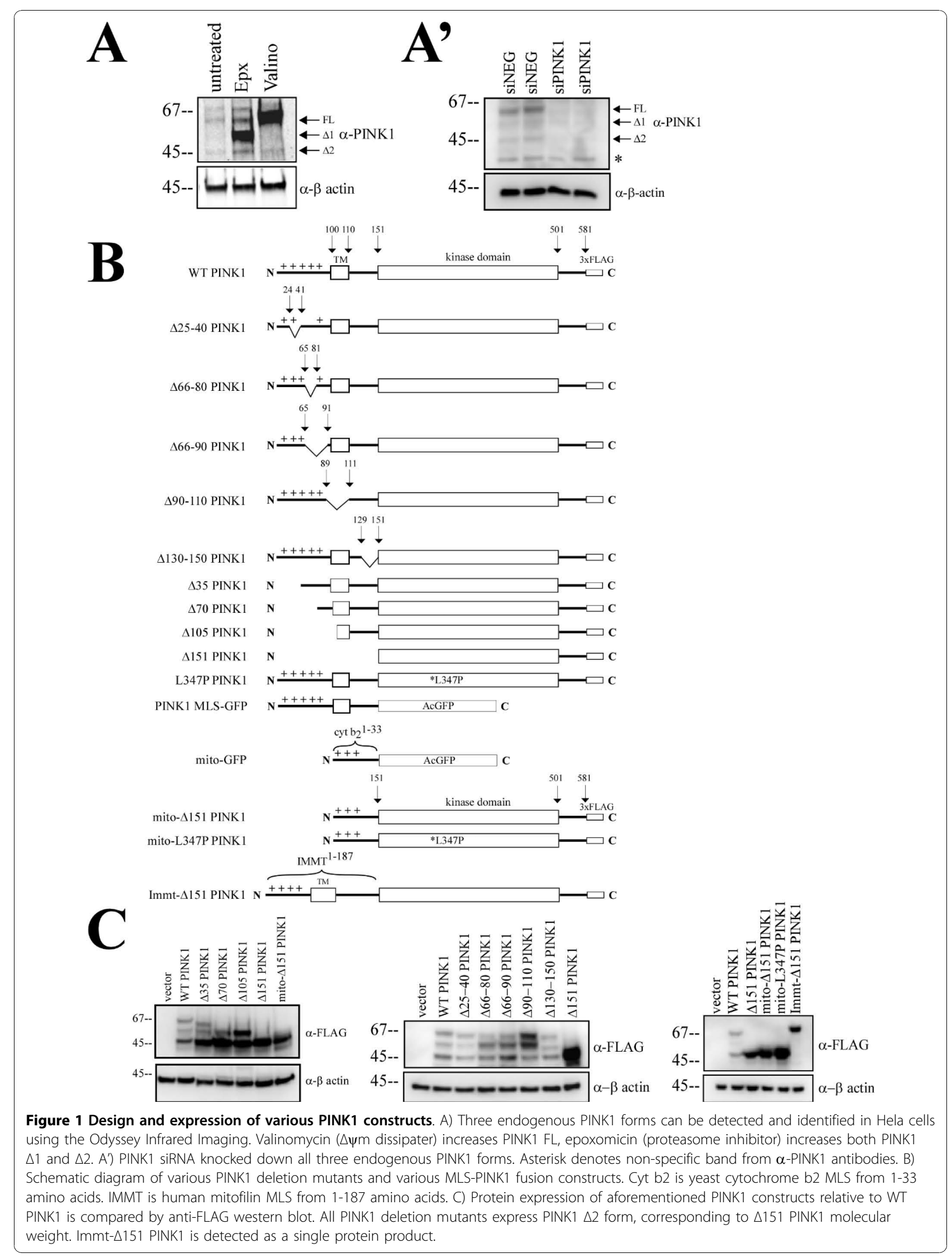


suggests that possible cleavage sites are within aa70-105 and aa105-151. This is similar to a recent publication using serial N-terminal deletion PINK1 constructs which suggested that the first cleavage site resides between aa91-101 [19], placing the putative cleavage site within the transmembrane domain. Since the disruption of N-terminal sequences may have affected mitochondrial targeting and cleavage, we also studied internal deletion mutants to map out the proteolytic sites in the PINK1 MLS (Figure 1B). By targeting the predicted cleavage sites in the PINK1 N-terminus, we truncated from aa25-40, aa66-80, aa66-90, aa90-110, and aa130-150. Unfortunately none of the internal deletions were able to abolish PINK1 cleavage (Figure 1C), illustrating the complexity of PINK1 MLS proteolysis. We did find that $\Delta 25-40$ PINK1 was consistent with $\Delta 35$ PINK1 in ruling out the cleavage site predicted at position 35 . Based on $\mathrm{N}$-terminal deletion mutants we predicted that a second cleavage site resides downstream of the transmembrane domain.

\section{PINK1 transmembrane and kinase domain determine PINK1 subcellular distribution}

As demonstrated before, WT PINK1 overexpression showed dual subcellular distribution with all three forms found in both mitochondrial and cytosolic fractions (Figure 2A). We asked how elements in the PINK1 structure can contribute to the mechanism behind PINK1 dual distribution. PINK1 protein contains three easily identifiable elements, an N-terminal MLS, a TM, and a C-terminal kinase domain. In general, the presence of a transmembrane domain in the MLS serves as a stop-transfer, or sorting signal, that prevents mitochondrial proteins from matrix import. We tested three most feasable hypotheses: 1) PINK1 TM serves as a stoptransfer signal, given that PINK1 is not found in the matrix and PINK1 mislocalized to the matrix compartment when the TM was deleted [19], 2) the cleavage after the transmembrane domain allows mitochondrial pool of PINK1 to become soluble, thus making it possible to redistribute to the cytosol, 3) the kinase domain interaction with Hsp90 in the cytosol prevents PINK1 from complete mitochondrial import, thus PINK1 adopts a topology where the kinase domain is exposed to the cytosolic face on the OMM.

We first tested the involvement of the TM in topology and dual distribution by using PINK1 MLS-GFP, where the PINK1 TM is intact but the C-terminal kinase domain is now replaced with GFP. We found that PINK1 MLS-GFP distributed only to the mitochondria and not the cytosol (Figure 2B and 3). This GFP fusion protein was protected from proteinase $\mathrm{K}$ digest, suggesting that it is likely localized inside the outer membrane (Figure 2B). As a control, we examined the mito-GFP protein by fractionation, using the cytochrome b2 MLS (1-33 aa). Mito-GFP also resisted proteinase $\mathrm{K}$ digest and was not found in the cytosol (Figure 2C). Combined, the data suggests the TM alone is not enough to lead to PINK1 topology with C-terminal portion of the protein facing the cytosol or cytosolic redistribution.

Next we examined our earlier hypothesis that the cleavage after the transmembrane domain allows tethered mitochondrial PINK1 to become cytosolic. Because we are unable to abolish the second PINK1 cleavage with our internal deletion mutants, we constructed and expressed Immt- $\Delta 151$ PINK1 fusion protein, one that contains the mitofilin MLS and the PINK1 kinase domain (Figure 1). Mitofilin is a mitochondrial inner membrane protein whose MLS includes a classical presequence followed by a TM, but not a proteolytic site downstream of the TM [24]. We found Immt- $\Delta 151$ PINK1 protein localized solely to the mitochondria and its sensitivity to proteinase $\mathrm{K}$ suggests an outer membrane topology (Figure 2D and 3). We reasoned that the lack of proteolysis after the TM prevents the release of Immt- $\Delta 151$ PINK1 from the mitochondria and it is very likely that Immt- $\Delta 151$ PINK1 is tethered to the outer membrane, similar to WT PINK1. The Immt- $\Delta 151$ PINK1 construct represents the first successful demonstration that we are able to eliminate the cytosolic pool of PINK1 while retain proper PINK1 mitochondrial topology.

We then asked whether the PINK1 kinase domain itself can confer tethered topology and cytosolic distribution. This time we deleted PINK1 MLS and fused cytochrome b2 MLS to the kinase domain. When we expressed mito- $\Delta 151$ PINK1, which now lacks a TM but retains the $\mathrm{C}$-terminal kinase domain, we found this protein distributed equally to the cytosol and the mitochondria. The mitochondrial fraction of mito- $\Delta 151$ PINK1 was protected from proteinase $\mathrm{K}$ digest, similar to matrix chaperone Hsp60 (Figure 2E and 3). We also examined the subcellular distribution of $\Delta 90-110$ PINK1, where the PINK1 TM is deleted. We found that $\Delta 90-110$ PINK1 predominantly localized to the mitochondrial fraction that is insensitive to proteinase treatment and a small fraction of cleaved $\Delta 90-110$ PINK1 was found in the cytosolic fraction (Figure 2F). Thus in the absence of a transmembrane domain, PINK1 has altered submitochondrial localization but some cytosolic redistribution remains. Taken all together, our data suggests that 1) the TM and the kinase domain are both needed for a tethered, cytosolic-facing, kinase domain topology and 2) PINK1 cytosolic redistribution requires both proteolysis after the TM and the kinase domain.

It was previously shown that PINK1 lacking MLS is mostly cytosolic although it can still interact with OMM or IMS proteins $[17,18]$. When we expressed $\Delta 151$ 


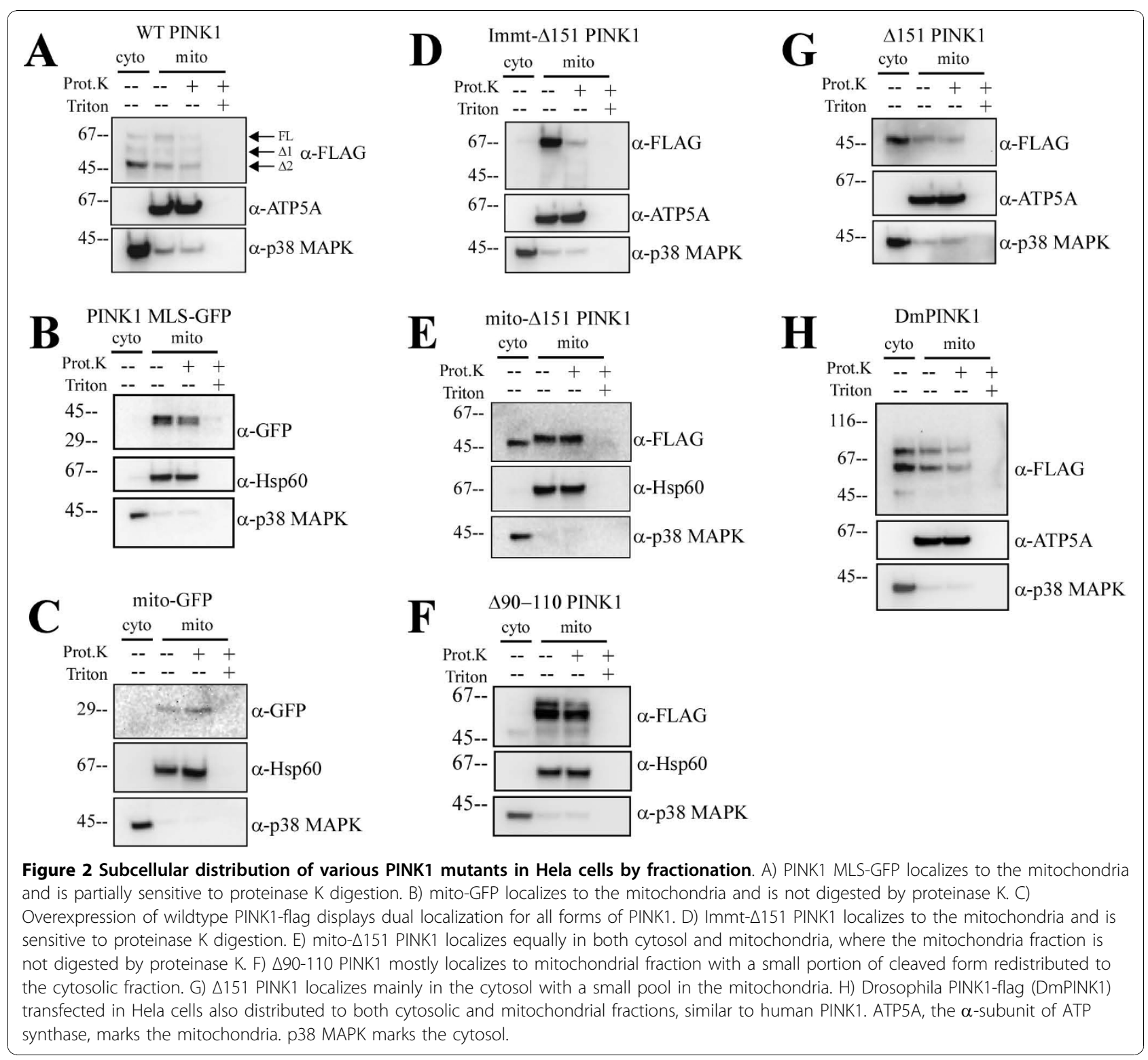

PINK1, lacking the N-terminal MLS, we found that this protein localized mostly to the cytosol, but some was still found in the mitochondrial fraction and co-localized with mitochondrial markers (Figure $2 \mathrm{G}$ and 3 ). It is likely that $\Delta 151$ PINK1 contains additional internal cryptic targeting signal because mitochondrially-localized $\Delta 151$ PINK1 was protected from proteinase K digest. Finally, we asked whether or not PINK1 dual distribution is evolutionarily conserved by examining the subcellular localization of drosophila PINK1. We found drosophila PINK1 in both cytosolic and mitochondrial fractions with two cleavage sites similar to the mammalian form (Figure 2H).

To further examine the idea that PINK1 kinase domain-Hsp90 interaction modulates mitochondrial entry of PINK1, we hypothesized that destabilizing the PINK1-Hsp90 interaction will increase PINK1 import into the mitochondria. We wanted to test the idea that the Hsp90 interaction is preventing PINK1 forward movement during mitochondrial import. We chose to use the PINK1 L347P mutation, a naturally occurring PD mutation with reduced Hsp90 interaction [13,25]. First we compared the subcellular localization between PINK1 WT and PINK1 L347P and found there was not observable difference in the cytosolic or mitochondrial distribution between the two proteins (Figure 4A). Even with a loss of Hsp90 binding, we reasoned that the intact transmembrane domain was enough to prevent PINK1 L347P from completely entering the mitochondria. Therefore, we constructed and expressed mito- 


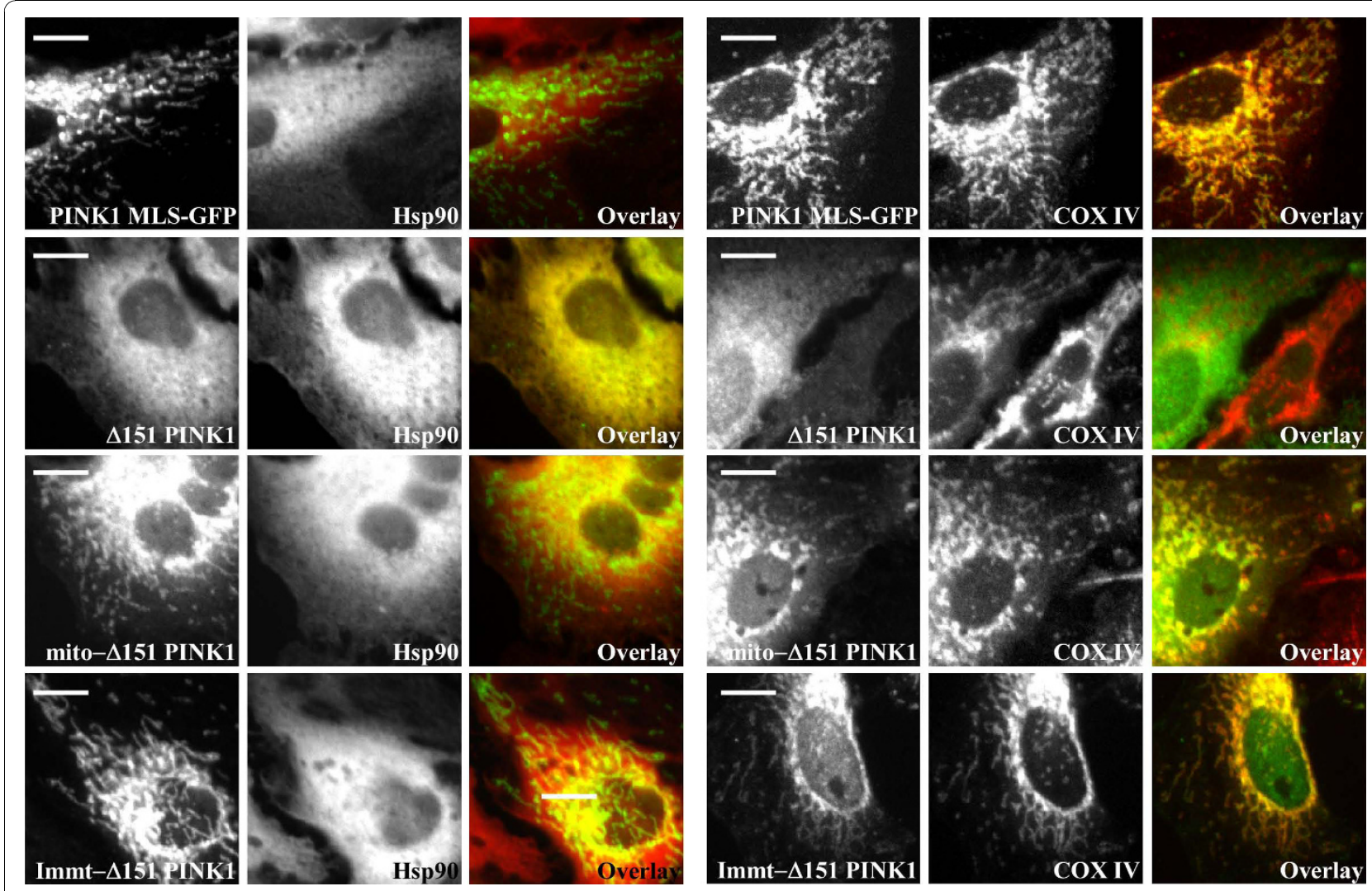

Figure 3 Immunofluorescence of PINK1-MLS GFP, $\Delta 151$ PINK1, mito- $\Delta 151$ PINK1, and Immt- $\Delta 151$ PINK1 in Hela cells. Cells transiently transfected with aforementioned constructs are also co-stained with either cytosol marker Hsp90 or mitochondria marker COX IV. Confocal images are analyzed with ImageJ and the yellow color in the overlay panels represents co-localization. Scale bar $=5 \mu \mathrm{m}$.

$\triangle 151$ PINK1 where we exchanged the PINK1 MLS with that of cytochrome b2 (1-33aa) to isolate the effect of TM out of the equation and to focus on Hsp90 interaction (Figure 4B). We compared the subcellular distribution of mito- $\Delta 151$ PINK1 in the absence and presence of Hsp90 inhibitor, 17-AAG (Figure 4B). We observed that in the presence of $17-\mathrm{AAG}$, mito- $\Delta 151$ PINK1 loses its cytosolic distribution with slight reduction in mitochondrial PINK1. We also noticed that the PINK1 protein sizes are slightly different between cytosol and mitochondria, although we are unsure of the explanation behind this size shift. It has been reported that matrix-localized PINK1 appears as a doublet either through post-translational modification or this size difference may arise from PINK1 having entered the mitochondria to have its MLS cleaved off by mitochondrial matrix protease [19]. In addition to the Hsp90 inhibitor experiment, we constructed mito-L347P PINK1 and compared its subcellular distribution to mito- $\Delta 151$ PINK1. When we compared the cytosol/mitochondria distribution between mito- $\Delta 151$ PINK1 and mito-L347P PINK1, there was significantly more $(\mathrm{p}=0.025)$ mitoL347P PINK1 $(1.226 \pm 0.086$, mean \pm SEM, $n=3)$ than mito- $\Delta 151$ PINK1 (0.888 \pm 0.044 , mean \pm SEM, $\mathrm{n}=3)$ in the mitochondria (Figure 4D-E). Lastly, we confirmed the Hsp90 interaction by co-immunoprecipitation and found a reduction in Hsp90 binding with mito-L347P PINK1 compared to $\Delta 151$ or mito- $\Delta 151$ PINK1 (Figure 4F). Full length L347P PINK1 also interacted less with Hsp90 compared to WT PINK1, and none of the GFP fusion proteins associated with Hsp90 (Figure 4F). These data suggest that the Hsp90 chaperone interaction on the cytosolic side can prevent PINK1 from further mitochondrial entry, consequentially leading to the release of PINK1 from the mitochondria once proteolysis removes PINK1 from the transmembrane anchor.

\section{Discussion}

As mentioned in the Introduction, both cytosolic and mitochondrial functions of PINK1 have been suggested. Elucidating the exact PINK1 subcellular localization will help us to understand these reported functions. The distribution of PINK1 in cells suggests that while a small percentage of PINK1 can be fully imported or associated with the mitochondria, the majority of PINK1 is 


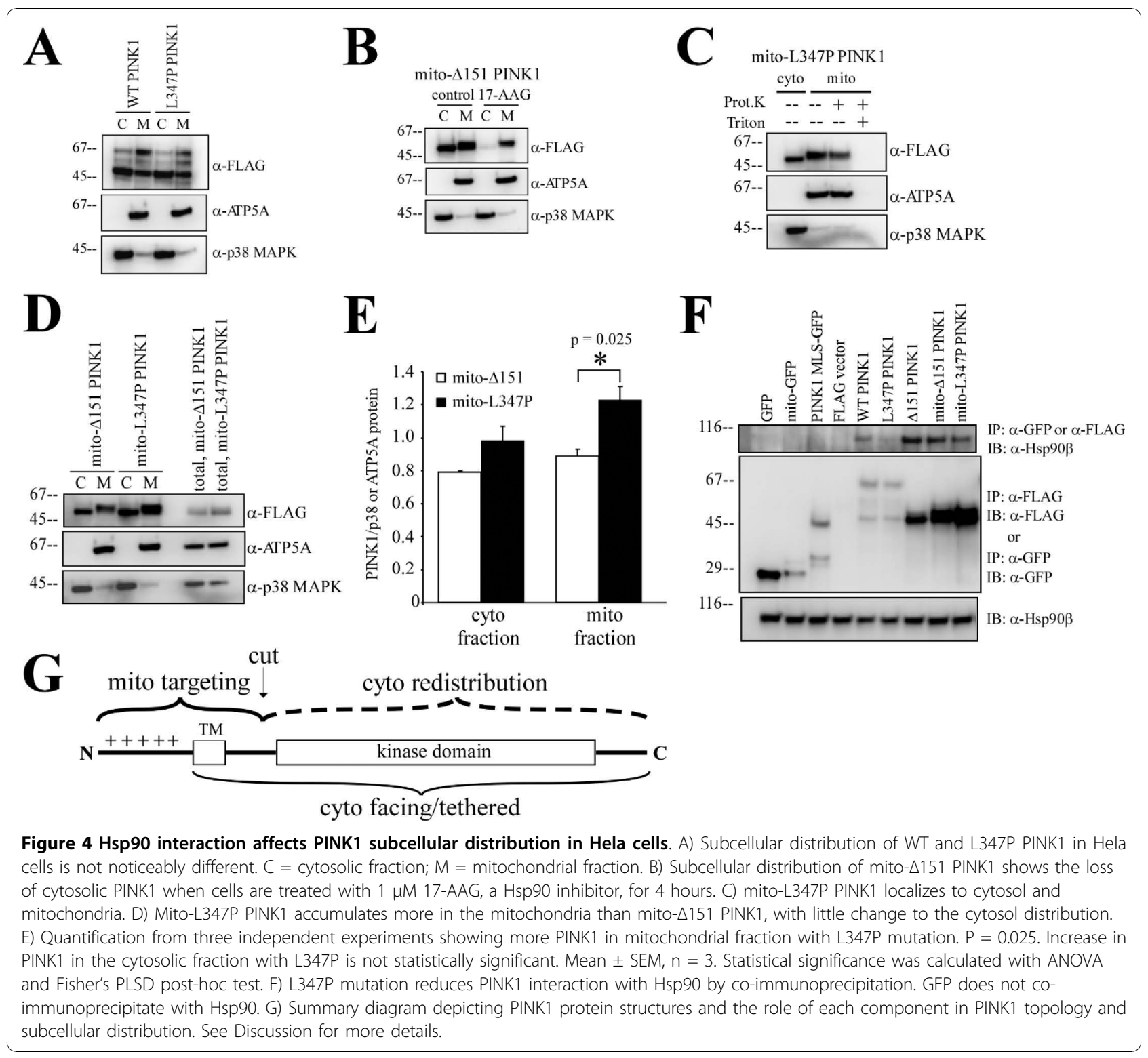

believed to reside in the cytosol. The demonstration that PINK1 contains a functional MLS and localizes within the mitochondria supports the hypothesis that PINK1 has a functional role in the mitochondria. While this functional role is unclear, several studies suggest a role of PINK1 in the mitochondrial fission/fusion pathway [26-28] and in mitophagy of damaged mitochondria [22,29-32]. Other compelling scientific data supports the hypothesis that PINK1 is also a cytosolic kinase. Strong evidence of a cytosolic degradation, cytosolic binding partners, and a protective function in the cytosol all point to a kinase protein with a dual localization and possibly two different functions, depending on the subcellular compartment. A major limitation in understanding the subcellular localization of PINK1 is the fact that many studies on PINK1 rely on PINK1 overexpression. Two challenges force researchers to utilize a heterologous overexpression system: the lack of a specific multipurpose antibody against PINK1 (the anti-PINK1 antibodies are only good for Western blot analysis) and the fact that the endogenous PINK1 expression level is very low. As we have demonstrated previously [12], properties of exogenous PINK1 are reflected by the endogenous PINK1, justifying that overexpressed PINK1 serves as a good model for the endogenous protein.

Unlike other mitochondrial proteins that localize exclusively to the mitochondria, mitochondrial proteins that adopt a cytosolic localization do so in a stimulusinduced fashion. With the exception of yeast fumarase and human PINK1, no other single gene-encoded, 
MLS-containing protein constitutively localizes to both the mitochondria and the cytosol, with the majority of the isoprotein residing in the cytosol. (At least we have not detected others so far.) In this paper, we investigated the important factors for PINK1 topology and dual localization and found three necessary components in the PINK1 protein-the transmembrane domain, the cleavage site after the TM, and the Hsp90 interaction (Figure 4G). We confirmed that the PINK1 MLS is responsible for mitochondrial localization and that two cleavage sites in the PINK1 MLS are responsible for generating PINK1 $\Delta 1$ and $\Delta 2$, present in both endogenous and exogenous PINK1. We attempted to map out the proteolytic sites by deleting the protein sequence encompassing the predicted cleavage sites. However, PINK1 continued to be cleaved into two products from the precursor. This could mean that we did not target the correct cleavage sites even though they are predicted by MitoPort or other prediction programs. PINK1 presequence cleavage might not follow the classical R-2/R-3/ $\mathrm{R}-10$ motif, where there are numerous examples [23,33]. Alternatively, it is thought that cleavage specificity of mitochondrial peptidases is less dependent on the primary protein sequence and more on the structural elements present in both the presequence as well as the mature protein [33]. Thus mutational or deletion studies (as we have done) will have variable results, including a lack of obvious effect on presequence cleavage. What is clear from our internal deletion study is that a second cleavage site is present after the transmembrane domain and this site plays an important role in PINK1 subcellular redistribution. Removal of this second cleavage site completely abolished cytosolic distribution of PINK1, as we showed with a noncleavable TM in mitofilin MLS. Because we are unable to abolish the cleavage of PINK1 MLS, we took advantage of the similarity between PINK1 MLS and mitofilin MLS to determine how presequence cleavage plays a role in PINK1 topology and distribution. Even though Immt- $\Delta 151$ PINK1 was not found in cytosol, it was digested by proteinase $\mathrm{K}$, similar to WT PINK1, suggesting that it is tethered to the outer surface of the mitochondria. We predict that if we substitute the PINK1 MLS with a bipartite presequence of an intermembrane space protein (ie cytochrome b2 [3]) then PINK1 would become soluble and redistribute to the cytosol.

When we addressed the role of the transmembrane domain, we confirmed the previous hypothesis that the transmembrane domain, acting as a stop-transfer signal, prevents forward import of PINK1 into the matrix. We demonstrated that in the absence of a transmembrane domain, either by deleting the PINK1 TM or by substituting PINK1 MLS with a matrix targeting signal, we were able to redirect mitochondrial PINK1 into proteinase-insensitive fraction. Thus the transmembrane domain is important, although not sufficient, for membrane tethering and cytosolic-facing topology.

We found that the PINK1 kinase domain, in conjunction with presequence cleavage, contributes to cytosolic redistribution of PINK1. Mitochondrial-targeted GFP (MLS-GFP and PINK1 MLS-GFP) were not found in the cytosol nor was GFP co-immunoprecipitated with Hsp90. When PINK1 kinase domain was present and co-immunoprecipitated with Hsp90, these recombinant proteins all showed dual subcellular distribution, except for IMMT- 151 PINK1 (as discussed previously). When we introduced natural PINK1 mutation L347P in the kinase domain, we not only disrupted the Hsp90-PINK1 interaction, we increased the mitochondrial PINK1 level, provided that a TM is absent. More PINK1 L347P mutant protein was found in the mitochondrial fraction compared to its wildtype counterpart. To explain why L347P PINK1 and mito-L347P PINK1 are found in the cytosol, we believe that a complete loss of Hsp90 interaction is necessary, as demonstrated by GFP proteins. In our co-immunoprecipitation experiment, L347P PINK1 and mito-L347P PINK1 showed significant reduction but not a $100 \%$ loss of Hsp90 interaction. This residual Hsp90 binding may account for the cytosolic redistribution. Of course, to completely eliminate PINK1-Hsp90 interaction will render PINK1 unstable and destine for rapid proteasome degradation. Importantly, we want to point out that decreased PINK1 retention in the cytosol consists of both accelerated degradation and increased PINK1 mitochondrial entry. When Hsp90 inhibitor, 17-AAG, was used in the experiment for Figure 4B, we did not see an increase in total mitochondrial PINK1 comparing untreated to 17-AAG-we actually saw a loss of signal. This is probably due to accelerated degradation and the loss of total PINK1. Thus we chose to complement the inhibitor data with the L347P mutation experiment-to avoid accelerating PINK1 degradation and other non-specific effects from 17-AAG, thereby to focus on how L347P mutation influences subcellular distribution. In that setting, mitochondrial PINK1 increased. Together, we believe that once PINK1 enters the mitochondria, PINK1 adopts a tethered topology because both the transmembrane domain and the kinase domain prevent PINK1 forward movement into the mitochondria. Subsequent proteolysis downstream of the transmembrane domain then releases PINK1 for retrograde movement while PINK1 kinase interacts with the Hsp90 chaperone.

As demonstrated by Zhou et al (2008), we find that PINK1 TM is required for kinase domain facing the cytosol. In addition, PINK1 kinase domain facing the cytosol also requires Hsp90 interaction and we believe it is the combined effects of TM and chaperone interaction that 
give mitochondrial PINK1 its proper topology. We have demonstrated that PINK1 $\Delta 2$ lacks the TM domain and thus its association with mitochondria must be through another mechanism. The question turns to whether or not PINK1 $\Delta 1$ is tethered to the mitochondrial membrane? We already know that this PINK1 cleaved form is rapidly degraded by the proteasome. Given the evidence that the first cleavage site might reside within the TM region, this suggests that PINK1 $\Delta 1$ might be loosely anchored or not anchored at all in its transient half-life.

\section{Conclusions}

In conclusion, the interaction of the kinase domain with Hsp90 plays a significant role in PINK1 topology and cytosolic redistribution. It is conceivable that Hsp90 binding to the PINK1 kinase domain is preventing the vectorial movement of PINK1 precursor protein during the entire import process. While PINK1 is targeted to the mitochondria, PINK1 function in the mitochondria is unclear. Published results show that loss of PINK1 can lead to mitochondrial dysfunction, but it is not clear that this is the result of losing mitochondrial PINK1 or cytosolic PINK1. Echoing a concern previously raised by Beilina et al (2005), the possibility that the cytosol contains mature PINK1 kinase challenges researchers to delineate how exactly PINK1 function links directly to mitochondrial functions. Embedded in this dual subcellular localization model is the proposal that PINK1 has compartment-specific functions, as was found for yeast fumarase. We believe that functional studies of PINK1 need to implement the experimental design of examining PINK1 function when it resides in only one subcellular compartment in order to tease apart PINK1 functional roles.

\section{Methods \\ cDNAs}

Mutant PINK1 cDNAs were amplified from wildtype human PINK1 cDNA via PCR-driven overlap extension [34] with the following primer pairs. The PCR product was then cloned into p3XFLAG-CMV14 (Sigma) flanked by EcoRI and BamHI restriction enzyme sites. $\triangle 25-40$ PINK1 (Forward 5' TTC ACG GGC AAG GTC CGC GGA GAG CGT 3'; Reverse 5'ACG CTC TCC GCG GAC CTT GCC CGT GAA 3'), $\triangle 66-80$ PINK1 (Forward 5' CTC GGG CTC CCT AAC TTG CAG CGG CAG TTC 3'; Reverse 5' GAA CTG CCG CTG CAA GTT AGG GAG CCC GAG 3'), $\triangle 66-90$ PINK1 (Forward 5' CTC GGG CTC CCT AAC GGC TGC GCG GGC CCT T 3'; Reverse 5' AAG GGC CCG CGC AGC CGT TAG GGA GCC CGA G 3'), $\triangle 90-110$ PINK1 (Forward 5' GTG GTG CGG GCC ATC GAG GAA AAA CAG 3'; Reverse 5' CTG TTT TTC CTC GAT GGC CCG CAC CAC 3'), 130-150 PINK1 (Forward 5' GTC AGG AGA TCC
AGT TTC GGC TGG AGG 3'; Reverse 5' CCT CCA GCC GAA ACT GGA TCT CCT GAC 3'), 151 PINK1 (Forward 5' ATT GAA TTC AAT GCG GCT GGA GGA GTA TCT G 3'; Reverse 5' ATA GGA TTA CAG GGC TGC CCT CCA TGA 3'), L347P PINK1 (Forward 5'CTG CTG CAG CTG CCG GAA GGC GTG GAC 3'; Reverse 5' GTC CAC GCC TTC CGG CAG CTG CAG CAG 3'). Drosophila PINK1 was cloned from Drosophila Gene Collection Clone GH20931 (Open Biosystems) with PCR (Forward 5' CAG AAG CTT ATG TCT GTG AGA CTG CTG AC 3'; Reverse 5' CAG GAT ATC AGC GCC ACC ACA TTC TGG A 3')

The starting ATG of GFP cDNA in pAcGFP-N1 plasmid (Clontech) was remove by PCR (Forward 5' CTT GGG ATC CAG TGA GCA AGG GCG CCG A 3'; Reverse 5' GTC GCG GCC GCT CAC TTG TAC AGC $\left.T C A T 3^{\prime}\right)$ and GFP was cloned back into the plasmid in BamHI and NotI sites; renamed plasmid GFP- $\triangle \mathrm{ATG}$. Mito-GFP was cloned by ligating annealed phosphorylated oligos of cytochrome b2 MLS into the EcoRI and BamHI site of GFP- $\triangle \mathrm{ATG}$ (Forward 5' phospho/AAT TCA TGC TAA AAT ACA AAC CTT TAC TAA AAA TCT CGA AGA ACT GTG AGG CTG CTA TCC TCA GAG CGT CTA AGA CTA GAT TGA ACA CAA TCC GCG CGT ACG GTT CTA CG 3'; Reverse 5' phospho/ GAT CCG TAG AAC CGT ACG CGC GGA TTG TGT TCA ATC TAG TCT TAG ACG CTC TGA GGA TAG CAG CCT CAC AGT TCT TCG AGA TTT TTA GTA AAG GTT TGT ATT TTA GCA TG 3'). To generate PINK1 MLS-GFP, PINK1 MLS was PCR amplified from WT PINK1 cDNA and cloned in the EcoRI and BamHI sites of GFP- $\triangle$ ATG by PCR (Forward 5' AAG AT TCA ATG GCG GTG CGA CAG GCG 3'; Reverse 5' ACT GGA TCC CGA AAG CCC TGC AAG C 3'). To generate mito- $\triangle 151$ PINK1, $\triangle 151$-flag was cloned into EcoRI and NotI of GFP- $\triangle$ ATG by PCR amplifying $\Delta 151$ PINK1 (Forward 5' ATT GAA TTC CGG CTG GAG GAG TAT CTG 3'; Reverse 5' ATT GCG GCC GCT CAC TAC TTG TCA TCG TCA T 3'). Then cyt b2 MLS was cloned into XhoI and EcoRI sites by PCR amplifying mito-GFP as template (Forward 5' ACC CTC GAG ATG CTA AAA TAC AAA CCT TTA C 3'; Reverse 5' AAA GAA TTC GGT AGA ACC GTA CGC GCG G 3'). To generate Immt- $\triangle 151$ PINK1, Immt MLS was PCR amplified and cloned into XhoI and EcoRI sites to replace cyt b2 MLS in mito- $\triangle 151$ PINK1 (Forward 5' AAA CTC GAG ATG CTG CGG GCC TGT C 3'; Reverse 5' AAT GAA TTC TGA AAG TGC AGG TGT $G G$ 3'). All plasmids were sequence verified. For PINK1 knockdown, siPINK1 oligos (Sense 5' GGA GAU CCA GGC AAU UUU UU 3'; Antisense 5' AAA AAU UGC CUG GAU CUC C 3') were purchased from Applied Biosystems/Ambion. Silencer Negative Control \#1 siRNA from Applied Biosystems/Ambion was used as 
scrambled siRNA control. Oligos were reverse transfected into Hela cells for three consecutive days with siPORT Amine Transfection Agent (Applied Biosystems/Ambion) according to manufacture protocol.

\section{Cell Culture and Transfection}

Hela CCL-2 cells were purchased from ATCC and cultured in DMEM complete media supplemented with $10 \%$ FBS and $1 \%$ penicillin/streptomycin. Transient transfection method with Lipofectamine 2000 was performed according to manufacturer's protocol (Invitrogen). Briefly, Hela CCL-2 cells were plated onto $60 \mathrm{~mm}^{2}$ tissue culture dishes at $90 \%$ confluency at the time of transfection. $2 \mu \mathrm{g}$ of cDNA was diluted in $250 \mu \mathrm{L}$ OPTI-MEM. $5 \mu \mathrm{L}$ of Lipofectamine 2000 was diluted in $250 \mu \mathrm{L}$ of OPTI-MEM. The mixture of cDNA and Lipofectamine 2000 was added to cells in OPTI-MEM. The transfection media was replaced by DMEM growth media six hours after transfection. Cells were subjected to experiments 48 hours following transfection.

\section{Co-Immunoprecipitation}

Co-IP experiments followed the methods described previously [35]. Briefly, cells were lysed in 1\% Triton X-100 buffer (20 mM HEPES [pH 7.5], $150 \mathrm{mM} \mathrm{NaCl}, 1 \%$ Triton X-100, 10\% glycerol, $1 \mathrm{mM}$ EDTA, protease inhibitor cocktail, and PMSF). Lysates were rotated for $1 \mathrm{hr}$ at $4^{\circ} \mathrm{C}$ then cleared by centrifugation at $13,000 \mathrm{~g}$ for 10 min at $4^{\circ} \mathrm{C}$. Equal protein amount was used for co-IP for all samples. Rabbit anti-FLAG (1:100) or anti-GFP $(1: 100)$ antibodies were used for immunoprecipitation at $4^{\circ} \mathrm{C}$ overnight. $30 \mu \mathrm{L}$ of Protein A/G PLUS agarose was added the next day, washed three times in $1 \%$ Triton X100 buffer, and resuspended in $2 \times$ sample buffer for SDS-HEPES PAGE (Pierce).

\section{Mitochondrial Isolation}

Mitochondria were isolated from Hela CCL-2 cells according to manufacturer's protocol (Pierce) with minor modifications. Briefly, the cells were trypsinized and harvested. A Dounce homogenizer was used to lyse the cells by 70 strokes. After removing the nuclear fraction, the crude supernatant was spun at 3,000 g for 20 minutes to pellet the intact mitochondria. The mitochondrial pellet was resuspended in IP buffer $(150 \mathrm{mM}$ $\mathrm{NaCl}, 50 \mathrm{mM}$ Tris- $\mathrm{HCl}, 0.5 \% \mathrm{NP}-40,0.5 \%$ sodium deoxycholate, $5 \mathrm{mM}$ EDTA, $0.25 \%$ SDS, $0.25 \mathrm{mM}$ PMSF and protease inhibitor cocktail) to collect mitochondrial proteins. For each fractionation, equal amounts of soluble cytosolic protein and mitochondrial protein were determined by BCA assay (Pierce). Proteins were resolved on SDS-HEPES PAGE.

\section{Proteinase $\mathrm{K}$ proteolysis assay}

Mitochondria were isolated by the mitochondrial isolation protocol described above. The mitochondrial pellet was resuspended in import buffer $(0.6 \mathrm{M}$ sorbitol, 50 $\mathrm{mM}$ HEPES, $50 \mathrm{mM} \mathrm{KCl}, 10 \mathrm{mM} \mathrm{MgCl}_{2}, 2 \mathrm{mM}$ $\mathrm{KH}_{2} \mathrm{PO}_{4}, \mathrm{pH} 7.0$ with $\mathrm{KOH}$ ) and aliquoted into three equal fractions. Final concentration of $50 \mu \mathrm{g} / \mathrm{mL}$ of proteinase K (Qiagen) was added to the appropriate sample tube with or without a final concentration of $1 \%$ Triton $\mathrm{X}-100$. Samples were incubated on ice for 30 minutes and the proteolysis was inhibited by the addition of PMSF and protease inhibitor cocktail. Then the samples were centrifuged at max speed for 5 minutes and the pellet was resuspended in IP buffer. Proteins were resolved on SDS-HEPES PAGE.

\section{Immunocytochemistry}

Transfected Hela CCL-2 cells were fixed in paraformaldehyde ( $4 \%$ for $10 \mathrm{~min}$ at room temperature) and then washed three times in $0.1 \%$ Triton X-100. Antigen retrieval was performed by incubating coverslips in $50 \mathrm{mM}$ Tris-buffered saline, $\mathrm{pH} 7.5$, at $95^{\circ} \mathrm{C}$ for $20 \mathrm{~min}$, followed by three washes in PBS. Nonspecific immunoreactivity was blocked with $10 \%$ goat serum. Cultures were incubated overnight at $4{ }^{\circ} \mathrm{C}$ in PBS containing a polyclonal FLAG (1:250 dilution) antibody and a monoclonal CoxIV or Hsp90 (1:250 dilution) antibody. Immunoreactivity to FLAG was amplified and detected using an Alexa 488 conjugate of a goat anti-rabbit IgG antibody and CoxIV and Hsp90 were amplified with Alexa 563 conjugate of a goat anti-mouse IgG antibody. The cells were imaged using a $150 \times, 1.35$ NA objective, and optical slices through the cultures were obtained using the 488 and $543 \mathrm{~nm}$ lines, respectively, of an Olympus DSU "fixed cell” Spinning Disk Confocal Microscope (Tokyo, Japan) at the Integrated Microscopy Core Facility at the University of Chicago. Images were analyzed with ImageJ (NIH).

\section{Western blot analysis}

Protein quantification was done using the BCA method (Pierce). Immobilon-P PVDF membrane was used in Western blotting (Millipore). After wet transfer, membrane was rinsed briefly with water. The membrane was blocked for 2 hours in blocking buffer (1XTBS containing $5 \%$ milk, and $0.1 \%$ tween-20). Appropriate primary antibodies were incubated for overnight in blocking buffer, and secondary antibodies were incubated in room temperature for 1 hour in blocking buffer. The membrane was then developed with ECL reagents (Milipore) and imaged with ChemiGenius Bio-Imaging system (Syngene). Optical density of protein signals were measured with ImageJ. Endogenous PINK1 was detected 
using Odyssey Infrared Imaging System (LI-COR Biosciences).

\section{Antibodies and Chemicals}

The following antibodies were purchased commercially. Anti-PINK1 (BC100-494) 1:1000 (Novus Biological), anti-FLAG 1:100 (Sigma), anti-FLAG M2 1:5000 (Sigma), anti-Hsp90 $\beta$ 1:250 (Santa Cruz), anti- $\beta$ actin 1:20,000 (Sigma), anti-p38 MAPK 1:2000 (Cell Signal Technologies), anti-CoxIV 1:200 (Invitrogen), antimouse IgG-HRP 1:20,000 (Promega), anti-goat IgG-HRP 1:1000 (Promega), anti-rabbit IgG HRP 1:10,000 (Promega), anti-ATP5A 1:10,000 (BD Biosciences), antiHsp60 1:10,000 (Cell Signal Technologies), anti-GFP 1:10,000 (Clontech). All chemicals are from Sigma, unless noted.

\section{Statistical Analysis}

Statistics were calculated with ANOVA followed by Fisher's PLSD post-hoc test for significance at 5\% with Statview software (SAS Institute, NC, USA).

\section{Abbreviations}

PINK1: PTEN-induced putative kinase 1; OMM: Outer mitochondrial membrane; MLS: Mitochondrial localization signal; GFP: Green fluorescent protein; FL: Full-length; TM: transmembrane; AA: amino acid.

\section{Acknowledgements}

We thank Drs. Lisa Won and Xiaoxi Zhuang for helpful discussions, Dr. Christine Labno for assistance with confocal microscopy, and Natasha Wadlington for technical assistance. This work was supported by generous fundings from American Parkinson Disease Association Post-doctoral fellowship (W.L), American Parkinson Disease Association Center for Advanced Research (U.J.K), and NIH R01 NS053919 (U.J.K).

\section{Authors' contributions}

WL co-designed the study, carried out all the experiments, performed data analysis, and co-wrote the manuscript. UJK co-designed the study, analyzed the data, and co-wrote the manuscript. Both authors read and approved the final manuscript.

Received: 26 April 2010 Accepted: 22 November 2010 Published: 22 November 2010

\section{References}

1. Chacinska A, Koehler CM, Milenkovic D, Lithgow T, Pfanner N: Importing mitochondrial proteins: machineries and mechanisms. Cell 2009, 138(4):628-644.

2. Matouschek A, Azem A, Ratliff K, Glick BS, Schmid K, Schatz G: Active unfolding of precursor proteins during mitochondrial protein import. Embo J 1997, 16(22):6727-6736.

3. Glick BS, Brandt A, Cunningham K, Muller S, Hallberg RL, Schatz G: Cytochromes $\mathrm{C} 1$ and $\mathrm{b} 2$ are sorted to the intermembrane space of yeast mitochondria by a stop-transfer mechanism. Cell 1992, 69(5):809-822.

4. Dagda RK, Barwacz CA, Cribbs JT, Strack S: Unfolding-resistant translocase targeting: a novel mechanism for outer mitochondrial membrane localization exemplified by the Bbeta2 regulatory subunit of protein phosphatase 2A. J Biol Chem 2005, 280(29):27375-27382.

5. Sass $E$, Karniely $S$, Pines $O$ : Folding of fumarase during mitochondrial import determines its dual targeting in yeast. J Biol Chem 2003, 278(46):45109-45116.

6. Sass E, Blachinsky E, Karniely S, Pines O: Mitochondrial and cytosolic isoforms of yeast fumarase are derivatives of a single translation product and have identical amino termini. J Biol Chem 2001, 276(49):46111-46117.

7. Beilina A, Van Der Brug M, Ahmad R, Kesavapany S, Miller DW, Petsko GA, Cookson MR: Mutations in PTEN-induced putative kinase 1 associated with recessive parkinsonism have differential effects on protein stability. Proc Natl Acad Sci USA 2005, 102(16):5703-5708

8. Silvestri L, Caputo V, Bellacchio E, Atorino L, Dallapiccola B, Valente EM, Casari G: Mitochondrial import and enzymatic activity of PINK1 mutants associated to recessive parkinsonism. Human Molecular Genetics 2005, 14(22):3477-3492.

9. Takatori $\mathrm{S}$, Ito $\mathrm{G}$, Iwatsubo T: Cytoplasmic localization and proteasomal degradation of N-terminally cleaved form of PINK1. Neurosci Lett 2008, 430(1):13-17

10. Pridgeon JW, Olzmann JA, Chin LS, Li L: PINK1 Protects against Oxidative Stress by Phosphorylating Mitochondrial Chaperone TRAP1. PLOS Biol 2007, 5(7):e172.

11. Petit A, Kawarai T, Paitel E, Sanjo N, Maj M, Scheid M, Chen F, Gu Y, Hasegawa H, Salehi-Rad S, et al: Wild-type PINK1 Prevents Basal and Induced Neuronal Apoptosis, a Protective Effect Abrogated by Parkinson Disease-related Mutations. J Biol Chem 2005, 280(40):34025-34032.

12. Lin W, Kang UJ: Characterization of PINK1 processing, stability, and subcellular localization. J Neurochem 2008, 106(1):464-474.

13. Weihofen A, Ostaszewski B, Minami Y, Selkoe DJ: Pink1 Parkinson mutations, the $\mathrm{Cdc} 37 / \mathrm{Hsp} 90$ chaperones and Parkin all influence the maturation or subcellular distribution of Pink1. Hum Mol Genet 2008, 17(4):602-616

14. Haque ME, Thomas KJ, D'Souza C, Callaghan S, Kitada T, Slack RS, Fraser P, Cookson MR, Tandon A, Park DS: Cytoplasmic Pink1 activity protects neurons from dopaminergic neurotoxin MPTP. Proc Natl Acad Sci USA 2008, 105(5):1716-1721

15. Tang $B$, Xiong H, Sun $\mathrm{P}$, Zhang $\mathrm{Y}$, Wang $D$, Hu Z, Zhu Z, Ma H, Pan Q, $\mathrm{Xia} J \mathrm{H}$, et al: Association of PINK1 and DJ-1 confers digenic inheritance of early-onset Parkinson's disease. Hum Mol Genet 2006, 15(11):1816-1825.

16. Xiong H, Wang D, Chen L, Choo YS, Ma H, Tang C, Xia K, Jiang W, Ronai Z, Zhuang $X$, et al: Parkin, PINK1, and DJ-1 form a ubiquitin E3 ligase complex promoting unfolded protein degradation. J Clin Invest 2009, 119(3):650-660.

17. Weihofen A, Thomas KJ, Ostaszewski BL, Cookson MR, Selkoe DJ: Pink1 Forms a Multiprotein Complex with Miro and Milton, Linking Pink1 Function to Mitochondrial Trafficking (dagger). Biochemistry 2009, 48(9):2045-52.

18. Plun-Favreau H, Klupsch K, Moisoi N, Gandhi S, Kjaer S, Frith D, Harvey K, Deas E, Harvey RJ, McDonald N, et al: The mitochondrial protease HtrA2 is regulated by Parkinson's disease-associated kinase PINK1. Nat Cell Biol 2007, 9(11):1243-1252.

19. Zhou C, Huang Y, Shao Y, May J, Prou D, Perier C, Dauer W, Schon EA, Przedborski S: The kinase domain of mitochondrial PINK1 faces the cytoplasm. Proc Natl Acad Sci USA 2008, 105(33):12022-12027.

20. Muqit MM, Abou-Sleiman PM, Saurin AT, Harvey K, Gandhi S, Deas E, Eaton S, Payne Smith MD, Venner K, Matilla A, et al: Altered cleavage and localization of PINK1 to aggresomes in the presence of proteasomal stress. J Neurochem 2006, 98(1):156-169.

21. Geisler S, Holmstrom KM, Treis A, Skujat D, Weber SS, Fiesel FC, Kahle PJ, Springer W: The PINK1/Parkin-mediated mitophagy is compromised by PD-associated mutations. Autophagy 2010, 6(7)

22. Narendra DP, Jin SM, Tanaka A, Suen DF, Gautier CA, Shen J, Cookson MR, Youle RJ: PINK1 is selectively stabilized on impaired mitochondria to activate Parkin. PLoS Biol 2010, 8(1):e1000298.

23. Hendrick JP, Hodges PE, Rosenberg LE: Survey of amino-terminal proteolytic cleavage sites in mitochondrial precursor proteins: leader peptides cleaved by two matrix proteases share a three-amino acid motif. Proc Natl Acad Sci USA 1989, 86(11):4056-4060.

24. John GB, Shang Y, Li L, Renken C, Mannella CA, Selker JM, Rangell L, Bennett MJ, Zha J: The mitochondrial inner membrane protein mitofilin controls cristae morphology. Mol Biol Cell 2005, 16(3):1543-1554.

25. Moriwaki Y, Kim YJ, Ido Y, Misawa H, Kawashima K, Endo S, Takahashi R: L347P PINK1 mutant that fails to bind to $\mathrm{Hsp} 90 / \mathrm{Cdc} 37$ chaperones is rapidly degraded in a proteasome-dependent manner. Neurosci Res 2008, 61(1):43-8.

26. Cui $M$, Tang $X$, Christian $W$, Yoon $Y$, Tieu K: Perturbations in mitochondrial dynamics induced by human mutant PINK1 can be 
rescued by the mitochondrial division inhibitor mdivi-1. J Biol Chem 2010, 285(15):11740-11752.

27. Yang Y, Ouyang Y, Yang L, Beal MF, McQuibban A, Vogel H, Lu B: Pink1 regulates mitochondrial dynamics through interaction with the fission/ fusion machinery. Proc Natl Acad Sci USA 2008, 105(19):7070-7075.

28. Poole AC, Thomas RE, Andrews LA, McBride HM, Whitworth AJ, Pallanck L: The PINK1/Parkin pathway regulates mitochondrial morphology. Proc Natl Acad Sci USA 2008, 105(5):1638-1643.

29. Geisler S, Holmstrom KM, Skujat D, Fiesel FC, Rothfuss OC, Kahle PJ, Springer W: PINK1/Parkin-mediated mitophagy is dependent on VDAC1 and p62/SQSTM1. Nat Cell Biol 2010, 12(2):119-131.

30. Michiorri S, Gelmetti V, Giarda E, Lombardi F, Romano F, Marongiu R, NeriniMolteni S, Sale P, Vago R, Arena G, et al: The Parkinson-associated protein PINK1 interacts with Beclin1 and promotes autophagy. Cell Death Differ 2010, 17(6):962-74.

31. Vives-Bauza C, Zhou C, Huang Y, Cui M, de Vries RL, Kim J, May J, Tocilescu MA, Liu W, Ko HS, et al: PINK1-dependent recruitment of Parkin to mitochondria in mitophagy. Proc Natl Acad Sci USA 2010, 107(1):378-383.

32. Dagda RK, Cherra SJ, Kulich SM, Tandon A, Park D, Chu CT: Loss of PINK1 function promotes mitophagy through effects on oxidative stress and mitochondrial fission. J Biol Chem 2009, 284(20):13843-13855.

33. Gakh O, Cavadini P, Isaya G: Mitochondrial processing peptidases. Biochim Biophys Acta 2002, 1592(1):63-77.

34. Heckman KL, Pease LR: Gene splicing and mutagenesis by PCR-driven overlap extension. Nat Protoc 2007, 2(4):924-932.

35. Shen J, Chen X, Hendershot L, Prywes R: ER stress regulation of ATF6 localization by dissociation of BiP/GRP78 binding and unmasking of Golgi localization signals. Dev Cell 2002, 3(1):99-111.

doi:10.1186/1471-2121-11-90

Cite this article as: Lin and Kang: Structural determinants of PINK1 topology and dual subcellular distribution. BMC Cell Biology 2010 11:90.

\section{Submit your next manuscript to BioMed Central and take full advantage of:}

- Convenient online submission

- Thorough peer review

- No space constraints or color figure charges

- Immediate publication on acceptance

- Inclusion in PubMed, CAS, Scopus and Google Scholar

- Research which is freely available for redistribution

Submit your manuscript at www.biomedcentral.com/submit
C Biomed Central 